\title{
INDEX
}

'the Abattoir', Bangkok 16, 20, 25

Abadie, Ricardo 121

abdominoplasty 43

abnormalities, correction of 44-47

academic research and discussion on international medical travel 2-3, 42, 145-146, 192

access to cosmetic surgery 4

Ackerman, Sara 115, 124, 136, 142, 153-154

actor-network theory (ANT) 71-72, 80-83

Adams, Josh 132

Adkins, Lisa 39

advertising of cosmetic surgery 87 , 130-133, 192

aesthetic judgements 117

aesthetic labour 82-83, 92-93, 110-114, 124-126

agents $98-105,127,138,158,167$, 180-181

tensions in dealing with 104-105

Aizura, Aren 153

American clinicians 118

American Society for Aesthetic Plastic Surgery (ASAPS) 32

Anderson, Ben 72-73, 84

Anderson, Warwick 72

anthropology 72

anti-ageing 44-45, 51

anxiety of patients 157

Appadurai, Arjun 27, 73-76, 80, 84-85

Arab Spring 167

Argentina 152-154

see also Buenos Aires

'armchair travelling' 131-132

assemblages 15, 27-28, 71-73, 79-85, 89, $99,129,193$

emergent temporality of 84

pluralised 85

Atkinson, Sarah 76-77 attentiveness 175

Australia 42-43, 46, 87, 189

Back, Les 173, 175-176

bariatric surgery 43

Baron-Faust, Rita 152

'beauty', use of the term 84

beauty systems and beauty capital 33-34, $37-40$

beautyscapes $84-85,150,192$

Beauvoir, Simone de 3

Beck, Ulrich 120

Belgium 43, 191

Ben Ali, Zine El Abidine 167

The Biggest Loser (TV show) 41

biomedicine 77-78

biopower 40

Bochaton, Audrey 81, 147-148

bodies subject to cosmetic surgery 27,40 , $47-56,61$

see also investment in one's own body

body capital 39, 45

body work 91, 112, 124, 126

Bolton, Sharon 157

'boobie birthdays' and 'boobie buddies' 140

Bordo, Susan 3, 33-36, 50

Botched-Up Bodies Abroad (TV show) 1, 70

Bourdieu, Pierre 38-39, 49

Bourke, Joanna 139

Braham, Mel 57

'brain drain' 15

Brazil 35, 75, 114, 118, 152

breast surgery $2,7,32,36,43-47,53-57$, $95,123-124,127,139,156,161,164$ problems with 56-57

British Association of Aesthetic Plastic Surgeons (BAAPS) 2, 32, 57

Brown, Wendy 37 


\section{Budapest 43}

Buenos Aires 79, 96, 152, 154-155

Bumrungrad international hospital, Bangkok 87, 129, 155-156

business failures 104, 106

Butler, Judith 37

Cameron, Keri 122, 125

care, logic of 68-71

'care convoys' 90, 97

'care transitions' 96

care work 90-96, 103, 106, 115-118, 124

Casanova, Erin 154

Casey, Victoria 95-96, 98

CE marks 56

celebrities 41

Chee, Heng Leng 73, 85, 106, 192

Chikanda, Abel 147

Chinese patients 13, 43-46, 124, 150-151, 179

choice, logic of 70-71, 120, 123

choices made by patients $68-71$

circumcision 32

cis-gendered women 34

cleanliness 48-49, 156-157

clinics 20-21, 87-88, 155

coding of data 12

Cohen, I. Glenn 106-107

Collier, Stephen 72-73

colonialism and postcolonialism 151, 157

community, membership of a 142-143, 193

compensation claims 191

competitiveness in the labour market 44

complications in the course of cosmetic surgery 189-190

conceptual framework for understanding cosmetic surgery 63

concierge services 15, 100-101

Connell, John 108, 147, 149

Connell, Raewyn 183

consultations, clinical 28, 33, 117-119, 123

consumerisation 64-65, 106, 115

conviviality $29,124,171-178$

tools for $175-176$

Cook, Peta 75, 79

Cooper, Charlotte 52

cosmetic surgery $1-5$

academic study of 2-3, 42

centres of expertise in 75

comparison of British and American narratives 35 considering the possibility of $8,122,165$, 192

definition of 3,31

framing in the media 42

opinions on 59

types of 13,43

cosmetic surgery tourism $4-5,8,13,28-31$, 64-81, 88-96, 127-128, 163, 177, 182, 192-193

antinomies in 64-71

attitudes towards $5,89,125,150$

dark side of 188

drivers of 4

employment in 180-181

expansion of 13,30

explanation and justification of 61

flows of 73-80

forms of 89-96

as an industry $28,86-89,180$

investment in 54-56

issues raised by 30

mapping of 4

personal and community-related aspects of 29

and personal identity 142

seen as a positive experience 128

websites on 76

'cosmeticness' 4, 65

cosmopolitanism 29-30, 171-172, 175-178

Costa Rica 115, 124, 153-154

costs of healthcare $4,54-55,76,88,99,111$, $122,181,190$

see also finance

Couldry, Nick 138

Crooks, Valerie 109

cross-border medical travel 147-148

Crush, Jonathan 147

cultural capital 38, 103-105

'cultural dopes' 60-61

Cunningham-Burley, Sarah 181

customer service 109

Czech Republic 43

Dalstrom, Matthew 98

data holding 12

data sources 4

Davis, Kathy 7, 33-36, 50

deaths through cosmetic surgery 188

'decapsulation' (Jansson) 140-141

defensiveness

of patients $5-6,37,61$

of surgeons 15 
Dholakia, Nikhilesh 155

diabetes, type-1 69-70

Diagnostic and Statistical Manual 34

disorienting experiences 157

distributed nature of care in cosmetic surgery 177

doctor-patient relationship 123, 129, 133

doctors at home and abroad 1

see also surgeons

'doctoring' (Mol) 69-70

Dovey, Kim 84

Drummond, Murray 184

Edmonds, Alexander 35, 39, 114

elective surgery $63-64,70-71,185$, 188-189

Elliott, Anthony 13, 89, 154, 159

embodied capital 41

emotional labour 15-18, 25, 92-95, $102-103,106,110,115,118,124,126$

emotions 75

empathy 175-176

empirical studies of cosmetic surgery, need for 192

employment in cosmetic surgery tourism 180-181

empowerment $35,41,120$

'enabling' function 63,88

enclaving 154

English language 157, 163, 170

enhancement surgery $32,44-47$

'entrepreneurialism' found in the public sector 112

ethical considerations 3, 8, 122, 193

see also self-improvement ethic

ethnoscapes 73-74, 77, 110

European Union 148, 191

Medical Services Directive 56

exoticisation 152

experimental treatments $4,78,181$

'expert patient' role 94, 125

Extreme Makeover (TV show) 36

Facebook 80-81, 105, 132-8

facilitators, full-service type and referral-service type 98

false consciousness 41

fashion 45

feminism and feminist analysis $3,5,7-8$, $32-38,41,82,127$

finance for cosmetic surgery, sources of 54,61 finanscapes 75-76

'First World care at Third World prices' claim 151

Foucault, Michel 33, 40

Fraser, Suzanne 83

friction with globalisation 79, 85

Gaddafi, Muammar 167-168

gender identity 34

gender reassignment surgery, use of 34,44 , 153,179

general practitioners (GPs) 14, 189

genital surgery 32

geographical knowledge, service users' lack of 168

geographies of international medical travel 145-151, 159

geography, imagined 29

Gibson, Margaret 127

Giddens, Anthony 120

Gillies, Harold 31

Gilroy, Paul 172-174

Gimlin, Debra 6, 35, 91-92, 164, 192

Glick Schiller, Nina 172

Glinos, Irene 146, 148

globalisation 2, 66-7, 71-73, 79, 85, 144-146, 174, 189

'bad' form of 144-146, 159

critical studies of 67

'glocalisation' 67

'god trick' 5

government

role of $88-89$

support for the industry 150

Graburn, Nelson 137

gracefully growing old 48-49

group experience 130-131

guarantees for surgery 191

guidebooks 76, 125

habitus 49

Harley Medical Group (HMG) 57-58

Hay, James 40

health

concept of 64,77

seen as a human right 1

health workers 108-119

aesthetic judgements about 117

healthcare systems

definition of 126

national 35 
heightened moments 137-138

Heyes, Cressida 36, 50-51

high-tech interventions 78

history of cosmetic surgery 31

Hochschild, Arlie 91, 109

Holliday, Ruth (co-author) 164

Hollway Wendy 5-6, 37, 61

'home', definition of 66

hope 77-79, 84, 181

The Hospital Group 57-8

hostessing 93

Huang, Shirlena 176-177

Huss-Ashmore, Rebecca 128-129

identity knowledges 7-8, 27

identity, restoration of $52-53$

identity work 120

ideoscapes 76-77, 109

image of cosmetic surgery 55,87

images used in the promotion of medical tourism 27

'immersion' in a new place 66

impression management 99

India 152-155

insurance, medical 13, 64, 88, 182, 191

interactions

'banal' 174

of cosmetic surgery tourists 129

intermediaries 88, 97-103, 187

hierarchies of $98-103$

international medical travel (IMT) 1-6,

$13,29,31,42-43,79,88-89,93-102$, 105-106, 108-112, 115, 119, 122-126, $144-148,154,173-178,181-182$, 188-191

academic literature on 2

casualties of 182

characterised as elite practice 2

dominant framings of 29

ethical dimensions of 3

growth in 188

marketing of 4

patient volumes $4,13,29$

range of 4

seen in context 6

stereotypes of 1

International Passenger Survey 13

international patient coordinators (IPCs) 95-99

definition of 98

Internet resources 80, 88, 128-133, 140

intersubjectivity of social interactions 51 interviews conducted for purposes of research 11-12, 37, 42

investment in one's own body 27,40 , $47-49,53-56,61,165$

InVivo software 12

Jansson, Andre 131-132, 140-142

Japan 46

jawbone reduction surgery 44,150

Jefferson, Tony 5-6, 37, 61

Jeffries, Sheila 33

Jeju Island 150

Jennings, Gayle 134

job satisfaction 115,181

Joint Commission International (JCI) 81, 89

Jones, Meredith (co-author) 40, 83

journeys made for purposes of cosmetic surgery $11,28-29,88,108,126$

unintended effects of 30

Kangas, Beth 78-79, 122, 182

kathoeys 156

Keogh Review 59

Kerr, E. Anne 181

Kingsbury, Paul 174

Kinsella, Sharon 46

Knoll, Eva-Maria 149

Korean Look 44-46, 49, 150

Lange, Patricia 134

language skills 157, 163, 180, 190

Lansley, Andrew 58-59

Lautier, Marc 83, 145, 147, 161, 167

Leder, Drew 184, 187

Lee, Jane Yeonjae 66

Libya and the Libyan conflict 30, 83-84, $125,160,166-169,174$

Linia (company) 57-58

literature from research on cosmetic surgery 31

'local globalness' 73

'logic of choice' and 'logic of care' (Mol) $68-71,77,91$

'logistical work' 122

lone travellers 136

low-cost travel 74-75, 88

McCann, Eugene 73

MacCannell, Dean 138

McDonald, Emily 79, 96, 123

McDowell, Linda 90 
McNay, Lois 141

'makeover culture' (Jones) 40

Maldives archipelago 149

male patient-consumers of cosmetic surgery $5,7-8,32,42-43,85,179$

Manderson, Lenore 146

mapping 84-85

Marcus, George 72

marketing and marketisation of cosmetic surgery $87-89$

'marriage cosmetics' 48

masculinity, hegemonic 183-187

Massey, Doreen 173

materialist approach to cosmetic surgery

$$
8,26-27
$$

Mazzaschi, Andrew 155

media representations 145

mediascapes 76-77, 83, 132

mediatisation 132

medical negligence 191

medical providers 98-99

medical records 189

medical tourism $65-67,74-77,122,128$, $144,153-154,160,176,182,189$

dangers of 111

geography of 144-154, 159

use of the term 145

medical travel facilitators (MTFs) 11-13, $15,28-29,63,73,81-86,97-108,111$, $122-125,127,134,138,142,180$

different levels of 99-101

as distinct from international patient coordinators 98-99

lack of medical qualifications 106, 108

roles of 102-106

types of 98-99

medicalisation of problems 114

Medicine and Healthcare products

Regulatory Agency (MHRA) 56-58

'meetingness' (Urry) 134

Mexico 75, 78, 148

middle class $38-41,48-49,164$

old and new 48

migrant workers 90, 93, 110

Miller, Daniel 54

Milligan, Christine 176-177

mind/body split 35

Mirivel, Julien 118

Mol, Annemarie 28, 68-69, 91, 120

momentum caused by tourists 79,85

moral panics 1,181

'moral triage' 124-125 muscles, working-class and middle-class types of 39

narratives, therapeutic $128,138,141$

national character 109, 153, 155

National Health Service (NHS) 55, 58-59, $87,92,181-182,185,189-190$

'burden' of cosmetic surgery on 14

national pride 118-119

Nederveen Pieterse, Jan 171

neoliberalism 40, 49, 61, 86-87, 151, 175, 181-182

Netherlands, the 35

networking 15, 27-28, 38, 80-82, 106, 128, 142,187

Noble, Greg 29, 172-174

nostalgia $51-52,61,153$

Novas, Carlos 77-78

Nowicka, Magdelena 174, 176, 178

Nuffield Council of Bioethics 192

nursing staff $28,109-110$

comparisons of cosmetic clinics with general hospitals made by 110

formal qualifications and special skills offered by 110

obesity 41

observation of surgery 15-16

octamethylcyclotetrasiloxane (D4) 58

Ong, Aihwa 72-73

online communities 142

open questions 6

options for treatment 78

orientalism 144-145, 151-159, 176, 178

Orlan 33

Ormond, Meghann 1, 74, 97, 120, 145-148, $153-154,178,181$

'othering' and 'otherness' 151, 174

Ouellette, Laurie 40

pain, experience of 139

passive role of patients 7

Paterson, Ian 188

pathologisation of patients 60-61

patient-centred care 115

patient-consumers 5, 64-66, 83-84, 179-182, 191

characteristics shared by $13-14$

work done by $28,93-94,119-125$

patient flows

direction of $144-148,159$

regional geography of $144-154,159$ 
'patienting' 119

patriarchy 33,41

Pattaya 156

Pauly Morgan, Kathryn 33

payment for healthcare see costs

Perfetto, Ralph 155

Petersen, Alan 78, 181

piercings 33

Pitts-Taylor, Victoria 82-83, 127, 141-142

placelessness 154-155, 159

Poland 43, 74, 155

Poly Implant Prosthèse (PIP) scandal 2, 56-60, 70, 75, 188, 191

Pordié, Laurent 66

post-essentialist analysis $127-128$

Poulter, Dan 59

power relations $7,12-13,118$

pregnancy, effects of 51-52

preparation for medical trips 122-123

see also research undertaken by patients prior to surgery

prestigious locations 123

private healthcare $14,76,115-117,120$, $146,151,153,156,180-182,191$

psychological pain 36

Puig de la Bellacasa, Maria 69

'Pygmalion complex' 114

quality of medical procedures and medical care 4

Rabinow, Paul 40

racism 173

rationing of healthcare 189

reconstructive surgery, use of 31-32, $44-47,113,189$

'recuperative comfort zones' 154

'reflexive modernity' 120

regionalising of healthcare 148-151

regulation of cosmetic surgery $61,88-89$, $188,191,193$

Reis, Michele 46

representation, problems of 37

reputation of the cosmetic surgery industry 87

research methodology $8-9,11$

research team for the present study attachments to disciplinary practices 7

differences within 12

make-up of 12

perspectives of 5 research undertaken by patients prior to surgery 129-130, 133-135, 179, 182

researching information for oneself 56 responsibilisation of patients 181-182, 187 retreats 136

risk management by patients $120-122$

risk of things going wrong $14,56-57,140$, 188-192

Robbins, Bruce 172

Roberts, Elizabeth FS 146

Rose, Nikolas 40, 77-78

Ruck, Nora 33

Said, Edward 151-152

Saka, Esen 72

Salazar, Noel 178

Sanchez Taylor, Jacqueline 164

satisfaction of patient-consumers with results of cosmetic surgery 14,51 , $134,158,182,189-190$

Savage, Mike 39

'scapes' 27, 73, 85

Scheper-Hughes, Nancy 146

secrecy which currently surrounds cosmetic surgery 31

self-actualisation 41

self-esteem 35, 39-40, 59-61, 114

self-governance 41

self-improvement ethic 188

Sengupta, Amit 152

Seoul and the Seoul Olympics 4, 74, 87, 150,179

Sinha, Shamser 173, 175-176

Skeggs, Beverley 38-39

Skountridaki, Lila 157

Smith-Morris, Carolyn 146

Snyder, Jeremy 105

Sobo, Eliza 66

social capital 38-39, 103-105

social class relations 38

social media platforms 14, 29, 80-81, 103, 128-143

social networks $80-81,125$

Solomon, Harris 98, 148, 153-155

Sothern, Matthew 120

South Africa 155

South Korea 13, 42-44, 46-48, 78, 110, $118,149-151$

see also Seoul

Soweto 155

Spitzack, Carole 33, 129

statistics on cosmetic surgery 32 
status hierarchies 91-92

stem cell tourism 78

stereotypes 1, 77, 153-154, 179

Stone, Sandy 34

Sulianti, Dian 97, 146-147

surgeons 111-119

accountability for shoddy work 191

care work undertaken by 115-118

comparison between those at home and those abroad 1, 55

creativity and variety of work 113 , 180

job satisfaction and patient-centred-ness of 115

patient-consumers' prior research on 14 , $129-130,133$

perspectives of $7-8,14-15,33,45-46$, 99, 103

professional identity and autonomy of 111-112

professional status of 82

relationships with MTFs 82, 111

see also consultations, clinical; doctorpatient relationship

Sutton, Barbara 154

tattooing 33, 39, 45

technoscapes 75

television programmes 1-2, 36-41, 55, 70, $76,125,166,181$

testimonials from patients $131-132$

Thailand 43, 109-110, 152-159, 163

theoretical framing for cosmetic surgery 27,63

Thompson, Charis 65

Throsby, Karen 50

'throwntogetherness' (Massey) 173-174

'tourism', use of the term 42

tourism work 92-94

'tourismness' 64, 93

'tourist', definition of 65-66

Toyota, Mika 67

trans people 34

Transform (company) 57-58

travelling companions 86, 94-97, 108

'treats' for the self 54

trustworthiness and truthfulness 133-135

Tsing, Anna 79

Tunisia 83-84, 124-125, 159-168, 173-174

Twigg, Julia 91 'uglification' of women's bodies 33

Urry, John 134

user-generated content 81,131

vaginal injuries 45,47

value, theory of 38

vanity $1-2,31-32,37,47,52$

Veijola, Soile 93

Velayutham, Selvaraj 177-178

Vertovec, Steven 174, 176, 178

'victim' model of cosmetic surgery 2

Viladrich, Anahi 152

visa restrictions 150

waiting times 6

Walton-Roberts, Margaret 108

Ward, Kevin 73

war-wounded soldiers 31

Weber, Brenda 40

websites, use of $29,76,130-133$

Wegenstein, Bernadette 33

weight-loss shows 41

weight-loss surgery 50

Weiler, Betty 134

Whittaker, Andrea 192

Wiegman, Robyn 5, 7, 27, 37

Wiles, Janine 176-177

Wilson, Ara 67

Wilson, Elizabeth 45

Wise, Amanda 172-173, 177-178

Wollaston, Sarah 2

women's looks 120-121

women's work 90

Wood, Stephen 84

Woodward, Ian 48

'word of mouse' 131

work

forms of 193

meaning of 126

sociology of 28

undertaken by patients themselves 28 , 93-94, 119-125

work practices, study of 89-92

working-class attitudes, practices and taste 38-39, 164

xenotransplantation 75

Yanhee hospital, Bangkok 155

Yeoh, Brenda 176-177

YouTube 134, 138 